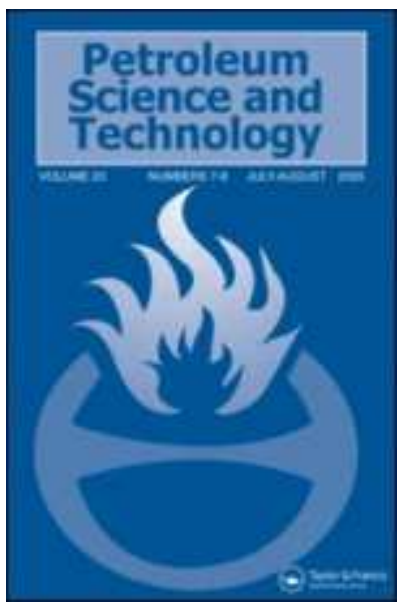

\title{
An explicit approximation of the Colebrook equation for fluid flow friction factor
}

\begin{tabular}{|c|c|}
\hline Journal: & Petroleum Science and Technology \\
\hline Manuscript ID: & LPET-2009-0351.R2 \\
\hline Manuscript Type: & Original Papers \\
\hline \multicolumn{2}{|l|}{$\begin{array}{r}\text { Date Submitted by the } \\
\text { Author: }\end{array}$} \\
\hline Complete List of Authors: & Brkic, Dejan; Ministry of Science and Technological Development \\
\hline Keywords: & $\begin{array}{l}\text { Hydraulic resistance, Colebrook equation, Approximate solution, } \\
\text { Lambert W-function, Flow friction }\end{array}$ \\
\hline
\end{tabular}

\section{S) ScholaroNE \\ Manuscript Central}




\author{
An explicit approximation of the Colebrook equation for fluid flow friction factor \\ Dejan Brkić, MSc in Petroleum Engineering, PhD Student \\ Ministry of Science and Technological Development, Đušina 7, 11000 Beograd, Serbia \\ Tel. +381642543668, fax: +381113243457, e-mail: dejanrgf@tesla.rcub.bg.ac.rs
}

\begin{abstract}
The Colebrook equation for determination of hydraulic resistances is implicit in fluid flow friction factor and hence it has to be approximately solved using iterative procedure or using some of the approximate explicit formulas which were developed by many authors. Here will be shown one approximation of the Colebrook equation based on Lambert W-function. Keywords: Hydraulic resistance; Colebrook equation; Approximate solution; Lambert Wfunction; Flow friction
\end{abstract}

Nomenclature

$\lambda$-Darcy friction factor (dimensionless)

Re-Reynolds number (dimensionless)

$\varepsilon$-Inner roughness of pipe $(\mathrm{m})$

D-Diameter of pipe (m)

X-argument of Lambert W-function

S-defined in text

\title{
1. Introduction
}

An integral part of the frictional pressure drop due to fluid flow in pipes involves the determination of hydraulic resistances, i.e. friction factor $(\lambda)$. An accurate determination of the 
pressure drop due to fluid flow in oil and gas wells is required for optimizing oil and gas production design calculations. Some of these calculations include developing tubing programs to maximize well deliverability, wellbore flow performance, sizing surface flow lines, and designing artificial lift installations (Farshad et al 2001). The design of oil or gas pipelines and networks is commonly presented through a series of numerical procedures and recommendations, and usually flow equations are recommended by the several authors according to common design and calculation practice (Coelho and Pinho 2007). Example of such equation can be here discussed Colebrook's (1).

The transition region between laminar and fully turbulent rough pipe flow was defined empirically on pipes that had a uniform roughness created by a coating of uniform sand covering the entire pipe interior. Measurements by Colebrook and White (1937) showed that pipes with non-uniform roughness did not display exactly the same transition curves. For commercial pipes Colebrook (1939) showed the transition region of turbulence could be described by:

$$
\frac{1}{\sqrt{\lambda}}=-2 \cdot \log \left(\frac{2.51}{\operatorname{Re} \cdot \sqrt{\lambda}}+\frac{\varepsilon}{3.71 \cdot \mathrm{D}}\right)
$$

Many researchers, as referred by Coelho and Pinho (2007) adopt a modification of the Colebrook-White equation (1), using the 2.825 constant instead of 2.51 .

This implicit equation was obviously too complex to be of practical use in the time when it was developed. It may be difficult for many to recall the time as recently as the 1970's when there 
were no personal computers or even calculators that could do much more than add or subtract. In that environment an implicit relationship such as Colebrook's (1), which was well-known then, was impractical and some simplification was essential. Rouse show that Colebrook equation is a reasonable and practically adequate solution and plotted chart based upon it (Moody 1944). In order to simplify this chart for ordinary engineering use, Moody (1944) adopted more convenient co-ordinates $\operatorname{Re}=\mathrm{f}(\lambda)$. In Rouse diagram friction factor $(\lambda)$ is implicit in both co-ordinates, i.e. $\operatorname{Re} \cdot \sqrt{\lambda}=\mathrm{f}(1 / \sqrt{\lambda})$. To be precise Rouse use primary axis as $\operatorname{Re} \cdot \sqrt{\lambda}=\mathrm{f}(1 / \sqrt{\lambda})$ and secondary as $\operatorname{Re}=f(\lambda)$, while Moody (1944) use only $\operatorname{Re}=f(\lambda)$. This graphical solution is the simplest but most approximate way to avoid trial-error or iterative solution to the implicit Colebrook relation. Today, equations such as Colebrook's can be easily solved very accurately in an iterative procedure using common software tools like MS Excel (Brkić 2010a). But, here will be shown one another way to solve Colebrook relation but no less accurate with Lambert W-function involved (Brkić 2010b). Among many today available explicit approximations to the implicit Colebrook equation (Moody 1947, Wood 1966, Eck 1973, Jain 1976, Swamee and Jain 1976, Churchill 1973, 1977, Chen 1979, Round 1980, Barr 1981, Zigrang and Sylvester 1982, Haaland 1983, Serghides 1984, Manadilli 1997, Romeo et al 2002, Sonnad and Goudar 2006, Rao and Kumar 2007, Buzzelli 2008), here will be shown another one based on Lambert W-function and its solution proposed by Barry et al (2000).

\section{On the Colebrook's equation for fluid flow friction factor}

For turbulent flow in rough pipes which is of greater practical interest, the Colebrook equation (1) is by far the most widely used correlation to calculate Darcy's friction factor $(\lambda)$. It relates the friction factor $(\lambda)$ to the Reynolds number $(\mathrm{Re})$ and relative pipe roughness ( $(\varepsilon / \mathrm{D})$. Before 1939., 
in which Colebrook's equation developed, for turbulent regime in smooth pipes widely was used Prandtl's equation also implicit in friction factor (2):

$$
\frac{1}{\sqrt{\lambda}}=2 \cdot \log \left(\frac{\operatorname{Re} \cdot \sqrt{\lambda}}{2.51}\right)=2 \cdot \log (\operatorname{Re} \cdot \sqrt{\lambda})-0.8
$$

Also, for turbulent regime in rough pipes widely was used von Karman's relation (3):

$$
\frac{1}{\sqrt{\lambda}}=2 \cdot \log \left(\frac{3.71 \cdot \mathrm{D}}{\varepsilon}\right)=1.74-2 \cdot \log \left(\frac{2 \cdot \varepsilon}{\mathrm{D}}\right)=1.14-2 \cdot \log \left(\frac{\varepsilon}{\mathrm{D}}\right)
$$

\author{
In that way transition curve between these two regimes does not exist. But experiments \\ conducted by Colebrook and White (1937) show that smooth curve between hydraulically \\ "smooth" regime and turbulent rough regime actually exist. From strictly mathematical point of \\ view, what Colebrook had done is incorrect, i.e. $\log (A+B) \neq \log (A)+\log (B)$, but physically this \\ relation gives good results, i.e. smoothing the contact between two lines (Figure 1).
}

Figure 1. Colebrook relation make transitional curve among hydraulically "smooth" regime and turbulent rough regime

Problem can be treated as inverse; according to logarithm's rules it is equally incorrect to split the Colebrook's relation into two pieces. 


\section{Transformation of the Colebrook's equation using Lambert W-function}

For real values of the argument $\mathrm{x}$, the $\mathrm{W}$-function has two branches, $\mathrm{W}_{0}$ (the principal branch) and $\mathrm{W}_{-1}$ (the negative branch). The range of the lower branch is $-1 \leq \mathrm{W}_{-1}$, while the upper branch $\mathrm{W}_{0}$ is divided into $-1 \leq \mathrm{W}_{0}^{-} \leq 0$ and $0 \leq \mathrm{W}_{0}^{+}$(Figure 2). $\mathrm{W}_{0}$ is referred to as principal branch of the Lambert W-function. The evolution of $\mathrm{W}$ function began with ideas began proposed by J.H. Lambert in 1758 and refined by L. Euler over the subsequent two decade (Gray 1978). Only $\mathrm{W}_{0}^{+}$ part of the principal branch of the Lambert W-function will be used for solution of our problem.

Figure 2. Real branches of Lambert W-function

Prandtl's equation (2) can be expressed in explicit form in exact mathematical way without any approximation involved (4):

$$
\frac{1}{\sqrt{\lambda}}=\frac{2}{\ln (10)} \mathrm{W}\left[\frac{\operatorname{Re} \cdot \ln (10)}{2 \cdot 2.51}\right]
$$

Similar transformation of Prandtl's equation (4) in explicit form is available in literature (Goudar, Sonnad 2003, 2007). Unfortunately, the Lambert W-function cannot be solved without some approximations. Further according to Brkić (2010b), after few logarithm and antilogarithm operation and used similar idea as original Colebrook's to unite Prandtl's and von Karman's relation, the Colebrook equation can be written in explicit form in exact mathematical way without any approximation involved (5). 


$$
\frac{1}{\sqrt{\lambda}}=-2 \cdot \log \left(10^{\frac{-1}{\ln (10)} \mathrm{W}\left[\frac{\mathrm{Re} \cdot \ln (10)}{5.02}\right]}+\frac{\varepsilon}{3.71 \cdot \mathrm{D}}\right)=-2 \cdot \log \left(\frac{5.02 \cdot \mathrm{W}\left[\frac{\mathrm{Re} \cdot \ln (10)}{5.02}\right]}{\operatorname{Re} \cdot \ln (10)}+\frac{\varepsilon}{3.71 \cdot \mathrm{D}}\right)
$$

Here shown procedures for solution of reformulated Lambert W-function easily can find application in professional software packages (Clamond 2009, Hayes 2005). No constraints in ranges for values of Reynolds number and relative roughness in comparisons to original Colebrook relation do not exist. Besides these two original reformulation of the Colebrook equation (Brkić 2010b) shown in this paper (5), in the paper of Moore (2006), another possible transformation of the Colebrook equation using Lambert W-function is shown. Similar equation is available in the paper of Nandakumar (2007) and in the papers of Sonnad and Goudar (2006, 2007). But relations shown in these papers have limitation in applicability for high values of Reynolds number and relative roughness because today available computers cannot operate with extremely large numbers (Sonnad and Goudar, 2004).

\section{Approximate solution of the Colebrook's equation based on the Lambert W-function}

Numerical approximations for solution of the Lambert W-function are also contained in some of the computer algebra packages. The Lambert function is implemented in many mathematical systems like Mathematica by Wolfram Research under the name ProductLog or Matlab by MathWorks under the name Lambert. Note that name "W" for Lambert function is not as old as the related function (Hayes 2005). The modern history of Lambert W-function began in the 
1980s, when a version of the function was built into the Maple computer-algebra system and given the name $\mathrm{W}$.

Formal solution of the Lambert W-function can be defined as (6):

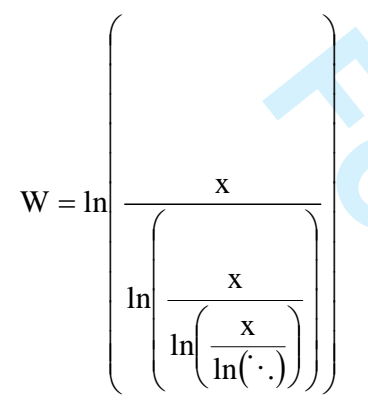

Boyd (1998) give his procedure for calculate of approximate value of Lambert W-function using so called "shifted" function. But here, approximate solution of transformed Colebrook equations (7) is done after procedure proposed by Barry et al (2000) (8).

$$
\frac{1}{\sqrt{\lambda}} \approx-2 \cdot \log \left(10^{-0.4343 \cdot \mathrm{S}}+\frac{\varepsilon}{3.71 \cdot \mathrm{D}}\right) \approx-2 \cdot \log \left(\frac{2.18 \cdot \mathrm{S}}{\mathrm{Re}}+\frac{\varepsilon}{3.71 \cdot \mathrm{D}}\right)
$$

Where $\mathrm{S}$ is (8):

$$
\mathrm{S} \approx \ln \frac{\operatorname{Re}}{1.816 \cdot \ln \left(\frac{1.1 \cdot \operatorname{Re}}{\ln (1+1.1 \cdot \mathrm{Re})}\right)}
$$

For more accurate procedure, but also more complex, readers can consult paper of Barry et al (2000). 


\section{Conclusion}

Main advantage of here shown transformed Colebrook equation (1) based on Lambert Wfunction written in explicit form in exact mathematical way without any approximation involved (5) is that accuracy of its solution depends only on accuracy of proposed procedure for solution of Lambert W-function. Here is shown one very accurate and simple approximation of the implicit Colebrook equation based on solution of the Lambert W-function after Barry et al (2000).

From the implicit Colebrook relation many explicit approximate formulas developed making possible a very accurate estimation of the friction factor without carrying out iterative calculus (Moody 1947, Wood 1966, Eck 1973, Jain 1976, Swamee and Jain 1976, Churchill 1973, 1977, Chen 1979, Round 1980, Barr 1981, Zigrang and Sylvester 1982, Haaland 1983, Serghides 1984, Manadilli 1997, Romeo et al 2002, Sonnad and Goudar 2006, Rao and Kumar 2007, Buzzelli 2008). Chen (1979), Schorle et al (1980), Gregory and Fogarasi (1985), Ouyang and Aziz (1996), Romeo et al (2002), Abdolahi et al (2007), Rao and Kumar (2007), Goudar and Sonnad (2007, 2008), Concha (2008), Brkić (2009), Yıldırım (2009), etc., in their papers made comparisons of in that time available approximations of Colebrook equation.

Here shown approximate solution of Colebrook equation (7), made maximal estimated relative error no more than $3.15 \%$ over the entire range of Reynolds number and relative roughness where original implicit Colebrook equation (1) can be used. This extreme value relative error is estimated for extreme low Reynolds number and relative roughness. Here shown approximation (7) is the most accurate for high values of Reynolds number and relative roughness among all 
other available approximations of the Colebrook relation. Estimated error in this region is below $0.002 \%$.

\section{References:}

Abdolahi, F., Mesbah, A., Boozarjomehry, R. B., and Svrcek, W. Y. (2007). The effect of major parameters on simulation results of gas pipelines. Int. J. Mech. Sci. 49 (8), 989-1000.

Barr, D.I.H. (1981). Solutions of the Colebrook-White function for resistance to uniform turbulent flow. Proc. Inst. Civil. Eng. 71 (2), 529-536.

Barry, D.A., Parlange, J.-Y., Li, L., Prommer, H., Cunningham, C.J., and Stagnitti, F. (2000). Analytical approximations for real values of the Lambert W-function. Math. Comput. Simulat. $53(1-2), 95-103$.

Boyd, J.P. (1998). Global approximations to the principal real-valued branch of the Lambert Wfunction. Appl. Math. Lett. 11 (6), 27-31.

Brkić, D. (2009). Comments on 'Settling velocities of particulate systems 15: Velocities in turbulent Newtonian flows'. Int. J. Miner. Process. 92 (3-4), 201-202.

Brkić, D. (2010a). Solution of the implicit Colebrook-White equation using Excel. Hydrocarb. Process. /in press, accepted 14 November 2008//

Brkić, D. (2010b). New reformulation of Colebrook-White equation for flow friction based on Lambert W-function. Hydrocarb. Process. /in press, accepted 23 July 2009/

Buzzelli, D. (2008). Calculating friction in one step. Machine Design 80 (12), 54-55.

Chen, N.H. (1979). An explicit equation for friction factor in pipes. Ind. Eng. Chem. Fundam. 18 (3), 296-297. 
Churchill, S.W. (1973). Empirical expressions for the shear stressing turbulent flow in commercial pipe. AIChE J. 19 (2), 375-376.

Churchill, S.W. (1977). Friction-factor equation spans all fluid flow regimes. Chem. Eng. 84 (24), 91-92.

Clamond, D. (2009). Efficient resolution of the Colebrook equation. Ind. Eng. Chem. Res. 48 (7), 3665-3671.

Coelho, P.M., and Pinho, C. (2007). Considerations about equations for steady state flow in natural gas pipelines. J. Braz. Soc. Mech. Sci. Eng. 29 (3), 262-273.

Colebrook, C.F. (1939). Turbulent flow in pipes with particular reference to the transition region between the smooth and rough pipe laws. J. Inst. Civil. Eng. (London) 11 (4), 133-156.

Colebrook, C.F., and White, C.M. (1937). Experiments with fluid friction in roughened pipes. Proc. Roy. Soc. Ser. A Math. Phys. Sci. 161 (906), 367-381.

Concha, F. (2008). Settling velocities of particulate systems 15: Velocities in turbulent Newtonian flows. Int. J. Miner. Process. 88 (3-4), 89-93.

Eck, B. (1973). Technische Stromungslehre. New York: Springer.

Farshad, F., Rieke, H., and Garber, J. (2001). New developments in surface roughness measurements, characterization, and modeling fluid flow in pipe. J. Petrol. Sci. Eng. 29 (2), 139150.

Goudar, C.T., and Sonnad, J.R. (2003). Explicit friction factor correlation for turbulent flow in smooth pipes. Ind. Eng. Chem. Res. 42 (12), 2878-2880.

Goudar, C.T., and Sonnad, J.R. (2007). Explicit friction factor correlation for turbulent flow in rough pipe. Hydrocarb. Process. 86 (4), 103-105. 
Gray, J.J. (1978). Johann Heinrich Lambert, mathematician and scientist, 1728 - 1777. Hist.

Math. 5 (1), 13-41.

Gregory, G. A., and Fogarasi, M. (1985). Alternate to standard friction factor equation. Oil Gas J. 83 (13), 120, 125-127.

Haaland, S.E. (1983). Simple and explicit formulas for friction factor in turbulent pipe flow. J. Fluid. Eng.-T. ASME 105 (1), 89-90.

Hayes, B. (2005). Why W? Am. Sci. 93 (2), 104-108.

Jain, A.K. (1976). Accurate explicit equation for friction factor. J. Hydraul. Div. ASCE 102 (HY5), 674-677.

Manadilli, G. (1997). Replace implicit equations with signomial functions. Chem. Eng. 104 (8), 129-130.

Moody, L.F. (1944). Friction factors for pipe flow. Trans. ASME 66 (8), 671-684.

Moody, L.F. (1947). An approximate formula for pipe friction factors. Trans. ASME 69 (12), 1005-1011.

More, A.A. (2006). Analytical solutions for the Colebrook and White equation and for pressure drop in ideal gas flow in pipes. Chem. Eng. Sci. 61 (16), 5515-5519.

Nandakumar, V. (2007). Analytical calculation of Fanning friction factor. Hydrocarb. Process. $86(1), 97-100$.

Ouyang, L.B, and Aziz, K. (1996). Steady-state gas flow in pipes. J. Petrol. Sci. Eng. 14 (3-4), $137-158$.

Rao, A. R., and Kumar, B. (2007). Friction factor for turbulent pipe flow. Preprint: Division of Mechanical Science, Civil Engineering, Indian Institute of Science, Bangalore, India, ID Code 9587, (from: http://eprints.iisc.ernet.in/9587/) 
Romeo, E., Royo, C., and Monzon, A. (2002). Improved explicit equation for estimation of the friction factor in rough and smooth pipes. Chem. Eng. J. 86 (3), 369-374.

Round, G.F. (1980). An explicit approximation for the friction factor-Reynolds number relation for rough and smooth pipes. Can. J. Chem. Eng. 58 (1), 122-123.

Schorle, B.J., Churchill, S.W., Shacham, M., and Chen, N.H. (1980). Comments on 'An explicit equation for friction factor in pipes'. Ind. Eng. Chem. Fundam. 19 (2), 228-230.

Serghides, T.K. (1984). Estimate friction factor accurately. Chem. Eng. 91 (5), 63-64.

Sonnad, J.R., and Goudar, C.T. (2004). Constraints for using Lambert W function-based explicit Colebrook-White equation. J. Hydraul. Eng.-ASCE 130 (9), 929-931.

Sonnad, J.R., and Goudar, C.T. (2006). Turbulent flow friction factor calculation using a mathematically exact alternative to the Colebrook-White equation. J. Hydraul. Eng.-ASCE 132 (8), 863-867.

Sonnad, J.R., and Goudar, C.T. (2007). Explicit reformulation of the Colebrook-White equation for turbulent flow friction factor calculation. Ind. Eng. Chem. Res. 46 (8), 2593-2600.

Swamee, P.K., and Jain, A.K. (1976). Explicit equations for pipe flow problems. J. Hydraul. Div. ASCE 102 (HY5), 657-664.

Wood, D.J. (1966). An explicit friction factor relationship. Civil. Eng. 36 (12), 60-61.

Yıldırım, G. (2009). Computer-based analysis of explicit approximations to the implicit

Colebrook-White equation in turbulent flow friction factor calculation. Adv. Eng. Softw. 40

(11), 1183-1190.

Zigrang, D.J., and Sylvester, N.D. (1982). Explicit approximations to the solution of Colebrook's friction factor equation. AIChE J. 28 (3), 514-515. 


\section{Figures}

Figure 1. Colebrook relation make transitional curve among hydraulically "smooth" regime and turbulent rough regime

Figure 2. Real branches of Lambert W-function 


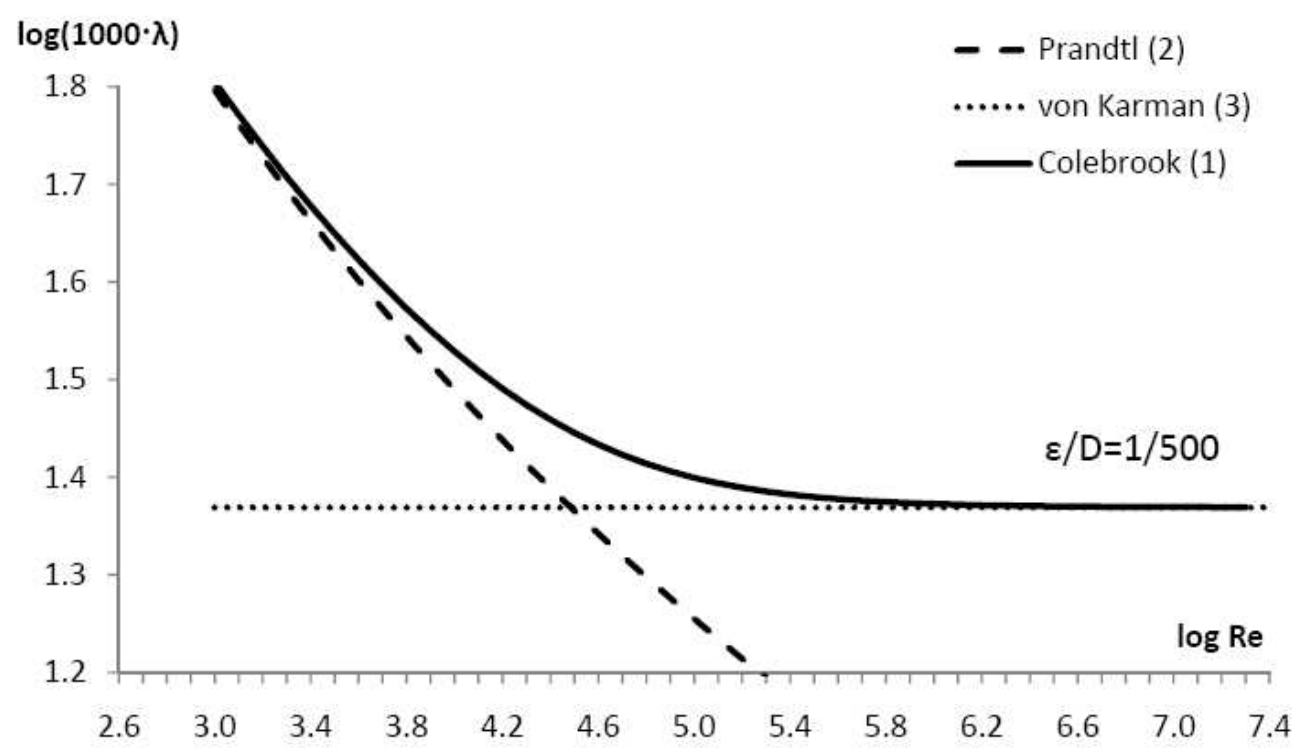

Colebrook relation make transitional curve among hydraulically "smooth" regime and turbulent rough regime

$60 \times 34 \mathrm{~mm}(300 \times 300 \mathrm{DPI})$ 


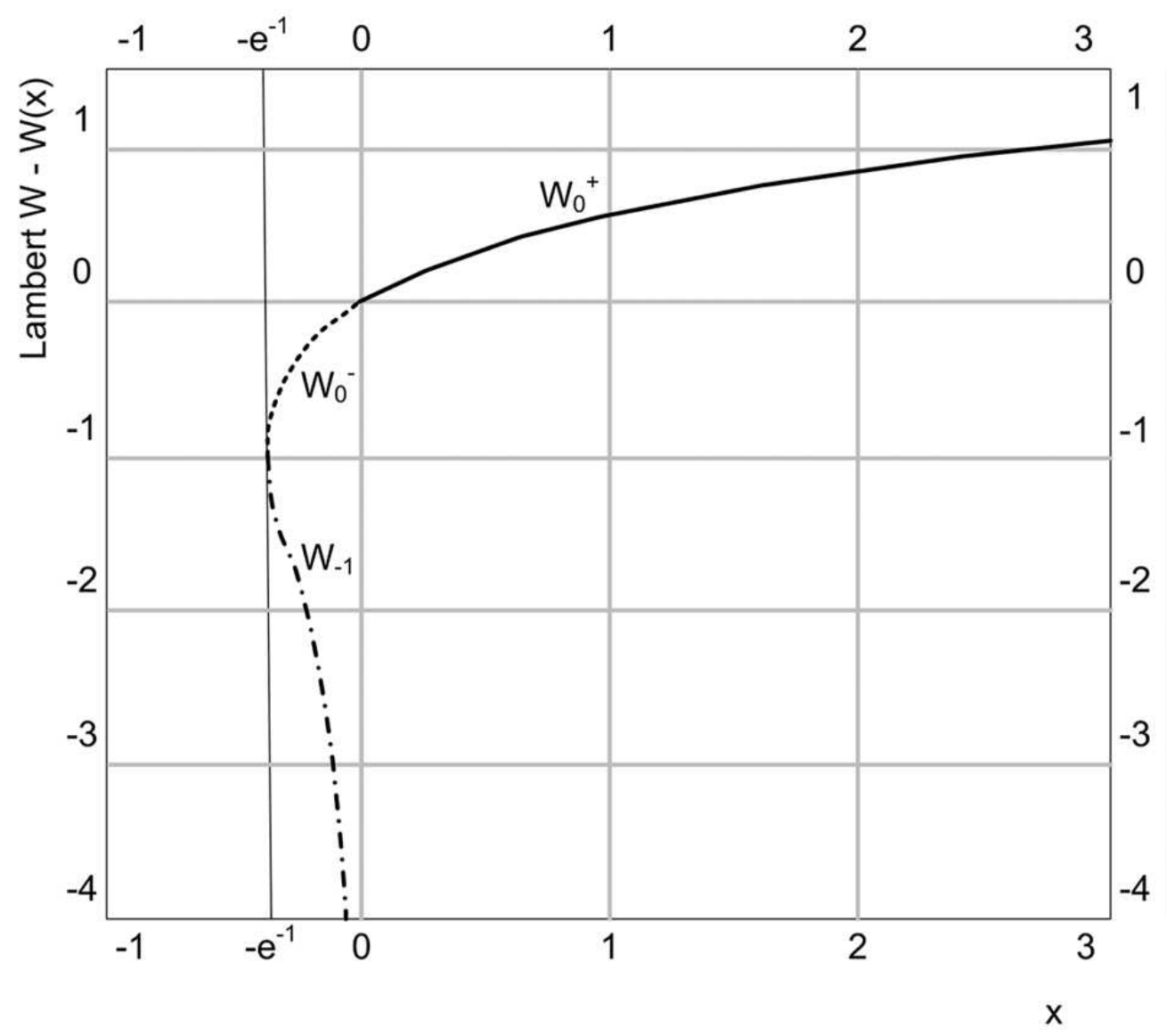

Real branches of Lambert W-function $80 \times 70 \mathrm{~mm}(300 \times 300$ DPI $)$ 